This report was prepared as an account of work sponsored by an agency of the United States Government. Neither the United States Government nor any agency thereof, nor any of their LBL -26130 employees, makes any warranty, express or implied, or assumes any legal liability or responsibility for the accuracy, completeness, or usefulness of any information, apparatus, product, or process disclosed, or represents that its use would not infringc privately owned rights. Reference herein to any specific commercial product, process, or service by trade name, trademark, manufacturer, or otherwise does not necessarily constitute or imply its endorsement, recommendation, or favoring by the United States Government or any agency thereof. The views and opinions of authors expressed herein do not necessarily state or reflect those of the United States Government or any agency thereof.

\title{
Raman Characterization of High Temperature Materials Using an Imaging Detector
}

\author{
Gerd M. Rosenblatt and D. Kirk Veirs \\ Lawrence Berkeley Laboratory \\ Materials and Chemical Sciences Division \\ 1 Cyclotron Road \\ Berkeley, CA 9472.0
}

(March 15, 1989)

for

Proceedings Sixth International Conference on High Temperature - Chemistry of Inorganic Materials, Gaithersburg, Maryland, April 1989, edited by J.W. Hastie.

This work was supported by the Director, Office of Energy Research, U.S. Department of Energy, at Lawerence Berkeley Laboratory under contract DE-AC-0376SF-00098. 


\begin{abstract}
The characterization of materials by Raman spectroscopy has been advanced by recent technological developments in light detectors. Imaging photomultipliertube detectors are now available that impart position information in two dimensions while retaining photon-counting sensitivity, effectively greatly reducing noise. The combination of sensitivity and reduced noise allows smaller amounts of material to be analyzed. The ability to observe small amounts of material when coupled with position information makes possible Raman characterization in which many spatial elements are analyzed simultaneously. Raman spectroscopy making use of these capabilities has been used, for instance, to analyze the phases present in carbon films and fibers and to map phase-transformed zones accompanying crack propagation in toughened zirconia ceramics.
\end{abstract}

Index Entries

Raman spectroscopy, irliging, carbon, zirconis, toughened 


\section{INTRODUCTION}

Understanding the chemistry and molecular architecture which underlies the physical properties of many materials requires chernical characterization of the material; i.e., characterization of the chemical composition, bonding and thermodynamic phase. With films, fibers, and many high-tech materials, sample volumes may be small and a sensitive chemical characterization technique is required. For instance, thin films of pure carbon exhibit a wide variety of electrical, mechanical and physical properties that depend upon the microscopic morphology which may encompass diamond, graphite and a wide range of amorphous or intermediate structures. Although the two common crystalline forms of pure carbon, graphite and diamond, are easily distinguished in bulk quantities by $\mathrm{X}$-ray diffraction, thin films of pure carbon are extremely difficult to characterize using $\mathrm{X}$-ray diffraction because of small signals and, in the case of amorphous films, the absence of long-range order.

In some cases macroscopic physical properties depend upon the chemical nature of the material changing across small distances. In these cases spatially-resolved chemical characterization is needed. For instance, ceramics which are strong but also unusually resistant to brittle fracture are produced by the controlled use of martensitic transformations, i.e., at least partly irreversible transformations between two solid phases having the same chemical composition. ${ }^{1}$ The anticipated increase in toughness has been demonstrated for cubic zirconia ceramics containing precipitates of tetragonal and monoclinic $\mathrm{ZrO}_{2}$. When a crack propagates in this material, the metastable tetragonal zirconia particles martensitically transform to the monoclinic phase in the high stress fields associated with the crack tip, forming a transformed zone surrounding the crack and crack tip. This transformation is thought to be a significant factor in the toughening of these materials. Theoretical approaches to understanding the fracture resistance of these materials indicate that toughening depends upon the zone width, the shape of the transformed zone around the crack tip, the extent of transformation within the zone, and the degree of irreversibility of the martensitic transformation. Understanding and optimizing the fracture resistance of such materials requires determining the phase composition in the transformed zone surrounding the crack with a spatial resolution of better than $50 \mu \mathrm{m}$. 
For both of these examples the distinguishing feature between the various forms of the materials is the difference in bonding (bond lengths, bond angles, and bond strengths) and not the chemical composition. This difference alters the fundamental atom-atom vibrations in the microcrystallites composing the material and can be detected using vibrational spectroseopy. Vibrational Raman spectroscopy is an optical technique in which scattered light is shifted in frequency by an energy equal to the energy difference between vibrational energy levels in the scattering material. The vibrational spectrum of a material is obtained by measuring the shift in energy of light scattered from a material illuminated with a monochromatic source. All materials have Raman-active vibrations with the one exception of a purely cubic phase.

The difficulty with Raman spectroscopy is that only a very small fraction of the incident light is Raman scattered. This means that it is not always easy to achieve reasonable signal:noise levels because of interference from other optical processes such as fluorescence or because of small signals in the case of small volumes. Recent advances in optical detectors have produced two-dimensional "imaging" detectors that have low noise and single-photon sensitivity. Such detectors, when carefully coupled with other recent advances in instrumentation, make it possible to apply Raman spectroscopy to materials problems which were formerly unaccessible to study because of the small sample volumes involved or the spatial resolution required.

In this paper we summarize some of the characteristics of a Raman apparatus built in our laboratory that utilizes an imaging photomultiplier tube for materials characterization. The salient capabilities are low noise, high sensitivity, and the capability to obtain one-dimensional profiles of chemical phase from a single illumination without moving the sample or scanning the spectrometer. Moving the sample allows subsequent profiles to be combined to produce a two-dimensional map of chemical phase. The characteristics and capabilities of the approach are illustrated by summaries of recent experiments in our laboratory on carbon films and fibers and on the phase-transformed zone surrounding cracks in phase- stabilized zirconia. 


\section{EXPERIMENTAL METHOD}

The major problem with the use of Raman scattering for the characterization of materials is that it is a weat phenomenon; the Raman cross-section for most materials is $10^{-31} \mathrm{~cm}^{2} \mathrm{sr}^{-1}$. This means that a $100 \mathrm{~mW}$ laser beam that illuminates a typical solid sample with $2 \times 10^{17}$ photons $\mathrm{s}^{-1}$ produces about $10^{10}$ Raman scattered photons $\mathrm{s}^{-1} \mathrm{sr}^{-1}$; these must be collected and spectrally dispersed to yield useful information. Even with bright light sources (lasers) and sensitive detectors (photon-counting photomultiplier tubes (PMTs)), long integration times are necessary to obtain good signal-to-noise ratios $(\mathrm{S} / \mathrm{N})$. Advances in multi-channel detectors, in particular the advent of the intensified photodiode array (IPDA), have increased the sensitivity of Raman spectroscopy. Using an IPDA coupled to a microscope, commercial Raman microprobe instruments hove demonstrated utility in investigating a number of materials problems which require a single point analysis of a small volume. ${ }^{2}$ At the present time, a new generation of sensitive detectors is available which produce data resolved in two position dimensions ( $X$ and $Y$ ) in contrast to the one-dimensional resolution $(X)$ of the IPDA. These new detectors, which include the imaging PMT and charge coupled device (CCD), also have less backgrousd and bence lower noise characteristics than the IPDA. These sensitive and low noise detectors can be used to extend the capabilities of Raman spectroscopy to investigate small amounts of material and to obtain multiplexed, spatially resolved, Raman spectra with a few microns spatial resolution.

The results presented here were obtained with a Raman spectroscopy apparatus incorporating an imaging PMT detector. This detector has several intrinsic features which allow a Raman spectrum to be obtained with both bigh sensitivity and multiplexed spatial resolution. First, the $25 \mathrm{~mm}$ diameter active region is partitioned into a $1024 \times 1024 \times \frac{\pi}{4}$ data array (the photoresponsive region is a disk that inscribes a $1024 \times 1024$ square). The detector is oriented so that one dimension (X) corresponds to the wavelength dispersion of the spectrometer and the second dimension $(Y)$ corresponds to distance along the sample. Second, the detector dark count which totals 30 counts per second (cps) at $-30^{\circ} \mathrm{C}$ is spread out over the $8 x$ $10^{5}$ active pixels yielding a dark count rate per pixel of just $4 \times 10^{-5}$ cps. Thus, detector dark count is virtually eliminated as a noise source. 
The Raman system has been described previously ${ }^{3}$ and only the main features and major changes will be emphasized. The apparatus consists of a $\mathrm{cw} \mathrm{Ar}{ }^{+}$laser usually operated at $488.0 \mathrm{~nm}$, focusing and collection optics, single spectrometer, imaging PMT detector, and microcomputer data acquisition. The Raman shifted light is isolated by a six-cavity interference filter which rejects the unshifted Rayleigh line by about six orders of magnitude while passing the region of interest with about 70\% transmission. The interference filter used for Rayleigh- scattering rejection adds an additional (rapidly varying) wavelength response to the (normally slowly varying) response of the spectrometer and detector. If accurate determinations of intensity ratios using an apparatus of the type described here are needed, the instrument response should be measured and used to correct the raw data.

Data are collected in two distinct modes depending upon whether or not a spatially-resolved profile is desired. When spatial resolution is not being sought the laser is focused to a spot of about $50 \mu \mathrm{m}$ diameter on the sample using a single lens. The scattered light is collected and focused onto the entrance slit of the spectrometer. The vertical dimension data are summed over a defined active region (typically ca. 100 rows) while wavelength dispersed information is retained. In this case, the detector dark count per wavelength channel is given by the number of rows which are active $(\sim 100)$ times the dark count per pixel, approximately $3 \times 10^{-3} \mathrm{cps}$ per wavelength channel. The $\mathrm{S} / \mathrm{N}$ of solid samples is always dominated by noise due to background signals from the sample. Many materials fluoresce strongly and special techniques not practical in our apparatus such as Fourier Transform Raman are needed to obtain their Raman spectra."

When spatial resolution is required the laser beam is expanded and then focused on the sample using a cylindrical lens. This produces a slit like illumination of $\mathbf{3 0}$ $\mu \mathrm{m} \times 2.5 \mathrm{~mm}$ which is imaged onto the entrance slit of the spectrometer. The cylindrical lens is rotated to align the image with the entrance slit. Each row in the detector array, corresponding to one spatial element along the illumination line at the sample, contains a unique Raman spectrum which can be analyzed to determine the chemical composition within that spatial element of the sample. Computer memory restrictions in our apparatus dictate that the data be acquired in a 256k position array. When desired this can be accumulated as a 256 wide $x$ 1024 high matrix where the spatial dimension retains the high resolution. Because 
approximately 800 rows of the detector data array are illuminated, this configuration simultaneously yields 800 Raman spectra from 800 consecutive spatial elements along the sample; from these a 800-position chemical profile can be derived. The integration times are typically less than 400 seconds with a maximum detected total count rate of about $10^{4} \mathrm{cps}$ which yields $5 \times 10^{3}$ counts over one row (256 channels wavelength channels) or an average of 20 counts per wavelength channel. The low noise characteristics of the detector allow such small signals to be obtained and analyzed; the detector contributes 0.01 total counts of background to the detected signal in one row over 400 seconds.

\section{RESULTS AND DISCUSSION}

\section{A. CARBON THIN FILMS AND FIBERS}

Graphite and diamond have quite different Raman spectra. Spear ${ }^{5}$ notes that Raman spectroscopy is the most reliable technique for distinguishing between crystalline diamond and other various forms of carbon and suggests that a working definition for "crystalline diamond material" in vapor deposited thin films include having a Raman spectrum typical of crystalline diamond (Fig. 1). Detailed analysis of the Raman spectrum of amorphous carbon, e.g. relative peak intensities, linewidths and absolute peak positions, can be used to estimate physical and chemical properties such as the average microcrystalline domain size and the $\mathrm{sp}^{2} / \mathrm{sp}^{3}$ bonding ratio. ${ }^{\circ}$

The Raman spectrum of carbon in the $1000 \mathrm{~cm}^{-1}$ to $2000 \mathrm{~cm}^{-1}$ range has been used to study the microscopic morphology of various forms of carbon. The data are corrected for the wavelength dependent intensity response which was calibrated using a tungsten intensity calibration lamp traceable to NBS. Single crystal graphite has a single, narrow Raman peak (the G-band) at $1575 \mathrm{~cm}^{-1}$ and a disorder induced peal: (D-band) at $1355 \mathrm{~cm}^{-1}$. Diamond's single, sharp Raman peak at 1332 $\mathrm{cm}^{-1}$ is distinct from the D-band of graphite, Fig. 1. The ratio of the integrated intensities of the D-band to the G-band has been correlated with average microcrystalline domain size, $\mathrm{L}_{a}$, as determined by $\mathrm{X}$-ray data ${ }^{7}$ and transmission electron microscopy. The observed increase in the relative intensity of the $\mathrm{D}$ (disorder) band relative to the $G$ (graphite) band with decreasing microcrystallite size may reflect 
the D-band intensity arising primarily from carbon atoms near the crystallite surface while the G-band arises from bulk-like carbon atoms in the interior volume. Linewidths of the D-band have also been correlated with $\mathrm{L}_{a}{ }^{8,9}$ Shifts of the G-band to lower frequencies have been related to the $\mathrm{sp}^{3}$ bonding fraction. ${ }^{10,11}$

Theoretical models of amorphous carbon with differing percentages of threefold $\left(\mathrm{sp}^{2}\right)$ and fourfold $\left(\mathrm{sp}^{3}\right)$ coordinated atoms indicate that the addition of fourfold coordinated atoms produces a gradual transition in the vibrational spectra to lower frequencies rather than a mixture of distinct features typical of graphite and diamond. ${ }^{10}$ Richter et al..$^{11}$ modeled the frequency of the G-band of graphite using force constants for both $\mathrm{sp}^{2}$ and $\mathrm{sp}^{3}$ bonded carbon atoms and found the shift in the frequency to lower energies to be essentially linear with increasing numbers of $\mathrm{sp}^{3}$ bonded carbon atoms.

Thin, sputtered carbon films $\sim 20-40$ nm thick used as protective overcoats on commercial $5 \frac{1}{2}$-inch computer hard disks have been investigated. The results are reported elsewhere ${ }^{6}$ and the salient features will be summarized here. Samples from two manufacturers designated as $\mathrm{K}$ and $\mathrm{L}$ were obtained; sample $\mathrm{K} 2\left(450^{\circ} \mathrm{C}\right.$ for $10 \mathrm{~h}$ ) and $155\left(350^{\circ} \mathrm{C}\right.$ for $\left.0.5 \mathrm{~h}\right)$ were annealed in vacuum. Raman spectra between 1000 and $2000 \mathrm{~cm}^{-1}$ were collected for four samples using $6.5 \mathrm{~mW}$ of laser power and a $1000 \mathrm{~s}$ integration time, Fig. 2. The spectra were fit to the sum of a linear background term and two damped oscillator line shapes. Each plot if Fig. 2 contains the corrected experimental data and three curves calculated from the fitted parameters. Two of the curves are calculated from the parameters representing the $G$ and $D$ bands individually and the third curve is the sum of the calculated background, $D$, and $G$ bands. The standard error of the fit, defined as the RMS average of the differences between the fit and the data divided by the data, zanged from 0.027 to 0.022 for all samples. Wavelength calibration was achieved by fitting $17 \mathrm{Ne}$ emission lines resulting in a $0.33 \mathrm{~cm}^{-1}$ standard error (less than one pixel).

From the fitting parameters one can calculate the peak position and linewidth of both the $\mathrm{D}$ and $\mathrm{G}$ bands, as well as the integrated intensity ratio, $I_{d} / I_{g}$. The results are shown in Table $I$. The observed ratios $I_{d} / I_{g}$ are considerably larger than one. Available literature data ${ }^{7}$ on the correlation of this ratio with domain size indicate that values close to or greater than one are always associated with graphite microcrystallite domains less than $5 \mathrm{~nm}$ across, but those data are limited to bulk 
samples having $I_{d} / I_{g} \leq 1$. Ratios considerably larger than one are routinely observed in thin films. ${ }^{8,11}$ The correlation with domain size in fine-grained films has not been measured, but measurements ${ }^{8}$ of $I_{d} / I_{g}$ as a function of annealing temperature suggest that for very swall grains ( $<2 \mathrm{~nm}$ ) $I_{d} / I_{g}$ increases with grain size (rather than decreases) to a maximum of 2.5 for $2 \mathrm{~nm} g$ ains, followed by a decrease in $I_{d} / I_{g}$ (similar to that observed with coarser carbon') as the grains grow further. It appears reasonable to postulate that the large $I_{d} / I_{\text {g }}$ ratios in Table I correlate with grains smaller than $5 \mathrm{~nm}$ and that the grain size increases with increased annealing temperature (and also with increasing $I_{d} / I_{g}$ ) in these thin, fine-grained films. From comparison of the peak positions and widths, the microcrystallites in L3 are larger $(>2 \mathrm{~nm})$ than those in the $\mathrm{K}$ series samples ( $<2 \mathrm{~nm}$ ) even though the $I_{d} / I_{g}$ ratios for L3 and $\mathrm{K} 1$ are nearly equal.

For the $\mathrm{K}$ series carbon films the Raman data suggest that these are composed of microcrystallites of less than $2 \mathrm{~nm}$ with $5 \% \mathrm{sp}^{3}$ bonds. Upon annealing the $\mathrm{sp}^{3}$ bond percentage drops essentially to $0 \%$ as the grains grow. In the L3 sample there are very few $\mathrm{sp}^{3}$ bonds and the microcrystals still are much smaller than $5 \mathrm{~nm}$, but probably larger than in the $K$ series. Diamond spectral peaks were not observed in any sample. However, the small grains and extensive $s^{3}$ eross-linking of the $K$ series films correlate with these having the "diamond-like" hardness required for the intended application.

Carbon fibers used to increase the strength of composite materials have much better separated D- and G-bands. Mechanical properties of polymer fibers have been related to their crystal quality as measured by $x$-ray diffraction. ${ }^{12}$ Graphite crystal quality is directly related to observables in the Raman spectra, namely the peak positions, linewidths, and intensity ratios of the 1355 (D) and 1575 (G) $\mathrm{cm}^{-1}$ peaks.

Fibers annealed at $2400^{\circ} \mathrm{C}, 2600^{\circ} \mathrm{C}$, and $2800^{\circ} \mathrm{C}$ have been examined and the $I_{d} / I_{g}$ ratio, line positions, and linewidths have been determined. The wavelength was calibrated using $\mathrm{Ne}$ and $\mathrm{Ar}$ discharge lamps and $\mathrm{Th}$ lines from a hollow cathode lamp to a standard error of $0.4 \mathrm{~cm}^{-1}$. The data were taken with $5 \mathrm{~mW}$ of 488.0 $\mathrm{nm}$ laser light incident on a fiber with an integration time of $500 \mathrm{~s}$ and a slit width of $121 \mu \mathrm{m}$. Representative Raman spectra are shown in Fig. 3. At laser powers above $200 \mathrm{~mW}$ the samples (a single carbon fiber) are destroyed by the focused 
laser beam.

The results are shown in Table II. The fibers have narrower $D$ and $G$ bands, smaller $I_{d} / I_{g}$ intensity ratios, a D-band shifted by ca. $40 \mathrm{~cm}^{-1}$ to lower frequency, and an unchanged $G$ (graphite) band position relative to the carbon films. These changes are consistent with much smaller surface:volume ratios, and larger microcrystallites, ca. $7-13 \mathrm{~nm}$, than the films. Heat treatment to $2600^{\circ} \mathrm{C}$ shifts both the D- and G-bands to higher frequencies by about $5 \mathrm{~cm}^{-1}$ and decreases their linewidths by about $20 \%$ as compared to the $2400^{\circ} \mathrm{C}$ samples. Further heat treatment to $2800^{\circ} \mathrm{C}$ does not significantly change the line positions or linewidths. In contrast, heat rreatment to $2800^{\circ} \mathrm{C}$ continues to change the intensity ratio. As discussed above, intensity ratios less than one have been correlated with the inverse of the domain size ${ }^{7}$ yielding a linear relation. Using this relation, the microcrystallite domain size of the carbon fiber samples appears to nearly double upon increasing the temperature from $2400^{\circ} \mathrm{C}$ to $2800^{\circ} \mathrm{C}$.

\section{B. TRANSFORMED ZONES IN PARTIALLY STABILIZED ZIRCONIA}

As mentioned in the introduction, elucidation of the toughening mechanism in high-tech phase-stabilized zirconia ceramics requires mapping the phase transformed zone surrounding cracks with a resolution of better than $\mathbf{5 0} \mu \mathrm{m}$. Under static loading a crack initialed in PSZ grows until a zone is formed around the crack tip where tetragonal phase has transformed martensitically into monoclinic phase. For the crack to grow further an increased static applied stress is necessary. The increased applied stress in turn produces a larger transformed zone and the crack again stops. Thus, under static loading, continously increasing applied loads are necessary for continued crack growth. However, under cyclic loading, cracks in PSZ grow without cessation. ${ }^{13}$ Thus failure is somewhat analogous to fatigue- crack growth in metals. The mechanism of crack growth under cyclic load is not fully understood and is being investigated.

Raman spectroscopy utilizing the two-dimensional detector and techniques described above can be used to map the transformed zone. ${ }^{14} A$ semi-automated experimental setup yields the fraction of monoclinic phase relative to the sum of the monoclinic and tetragonal phases, determined from the Raman spectrum, as a function of distance across the surface of the specimen. First, a one-dimensional 
chemical profile oi 800 elements is determined by analysis of the data resulting from a single 400 s slit-lige illumination of a line across the sample. Second, the sample is translated a known amount perpendicular to the slit-like illumination and another one-dimensional chemical profile is determined. This process is rapeated automatically and successive profiles are then combined to produce a map of the phase distribution.

The partially stabilized zircania used for this study is cornpised of $50 \mu \mathrm{m}$ grains of cubic-phase zirconia with lenticular precipitstes of tetragonal and monoclinie: phases with a maximum dimension of $300 \mathrm{~nm}$. The tetragonal-pliese precipitates, which otherwise would transform martensitically to mone:linic at room temperature, are stabilized by the addition of 9 mol\% MgO. 1 se presipitstes form $25-40 \%$ by volume of the cubic grains.

A Raman spectrum typical of PSZ used in these studies is shown in Fig. 4. To calculate a phase profile, the fraction of monoclinic is computed usiag the integrated intensities (minus the background) of the 131 and $192 \mathrm{~cm}^{-1}$ peaks for the monoclinic phase and the integrated intensity (minus the backgrour d) of the $264 \mathrm{~cm}^{-1}$ peak for the tetragonal phase. The monoclinic and teirozonal phuses are assumed to produce the same relative Raman intenrities; this assumption is consistent with literature relative Raman intensities's and yields results that are not inconsistent (within the large, perhaps a factor of two, uncertainty in the X-ray determination) with the composition of samples determined by X-ray diffractrion. The relative Raman response of the two phases affects the scale, but not the topography cf the compositional maps that are determined.

The application of spatially-resolved Raman spectroscopy to eracks grown in peak-toughened PSZ is described in detail in Ref. 14. That report describes results on a crack grown using a constant cyclic load. In subsequent work we have in"estigated a crack grown with a varying amplitude cyclie load, during fatigue-crack growth in a peak-toughness $\mathrm{MgO}$-PSZ sample $3 \mathrm{~m} n$ thick. ${ }^{16}$ The crack-growth rate exhibits sharp changes which depend upon changes in the magnitude of the load. Because the crack-growth rate depends upon tine transformed zone, detailed measurements of the transformed zone are required in order to understand the behavior of the rate of crack growth and the implications for failate mechanisms under varying load conditions. 
The specimen was cyclically loaded at a load ratio of $0.1\left(\mathrm{~K}_{\min }=0.1 \mathrm{I}_{\text {maxi }}\right.$ where $K_{\min }$ and $K_{\max }$ are, respectively, the minimum and maximum stress intensities in a given fatigue cycle; $K_{\max }$ and $\mathrm{K}_{\min }$ are changed together in varying-load experiments) and frequency of $50 \mathrm{~Hz}$ in a high-resolution, computer-controlled electro-servohydralic testing machine. ${ }^{16}$ Crack-growth rates, $\mathrm{dA} / \mathrm{dN}$, were determined during computer controlled applied stresses over a range of $\Delta \mathrm{K}\left(\Delta \mathrm{K}=\mathrm{K}_{\text {mex }}\right.$ $\left.-K_{\min }\right)$. Fig. 5 shows the applied stress and the crack-growth rate as a function of crack extension. A constant high cyclic load is first applied $(\Delta \mathrm{K}=9.5 \mathrm{MPa} \sqrt{m})$ and the crack-growth rate increases toward a steady state value. On reducing the cyclic loads somewhat (to $\Delta \mathrm{K}=8.5 \mathrm{MPa} \sqrt{\mathrm{m}}$ ), a marked transient retardation in crack velocity is seen followed by a gradual increase in crack-growth rate toward a (new) steady- state velocity. A significant retardation is seen following a single tensile overload to a $\mathrm{K}_{\max }$ of $12.3 \mathrm{MPa} \sqrt{\mathrm{m}}$ ) (the fracture toughness of this material is $\left.\mathrm{K}_{c}=16.0 \mathrm{MPa} \sqrt{m}\right)$ ).

The Raman map of the phase distribution of this sample, Fig. 6, is constructed from 65 chemical profiles taken perpendicular to the crack every $125 \mu \mathrm{m}$. A laser power of $150 \mathrm{~mW}$ and an integration time of $200 \mathrm{~s}$ per profile was used. Calculation of the chemical profile from each illumination takes 132 s so the total time for data collection and analysis was 6 hours $((200 s+132 s) \times 65)$. Further development of the data collection and computation algorithm now allow this same profile to be collected in less than 4 hours (200 s $\times 65$ ). The count rate was $32000 \mathrm{cps}$ and the average count per pixel was 24 . The transformation zone shows considerable changes in the zone width and extent of transformation which are related to the applied stress.

The crack-growth rate can be understood qualitatively by considering the size of the transformed zone and the magnitude of the applied stress. If the transformed zone is large the rate of crack growth (under a given cyclic applied stress) will be relatively small. Conversely, if the transformed zone is small, the crack- growth rate will be relatively large. The size of the transformed zone surrounding the crack tip is dependent upon the stress that has bet. $\mathrm{applied.} \mathrm{When} \mathrm{the} \mathrm{applied} \mathrm{stress} \mathrm{is}$ lowered from $\Delta \mathrm{K}=9.5 \mathrm{MPa} \sqrt{m}$ to $\Delta \mathrm{K}=8.5 \mathrm{MPa} \sqrt{m}$ the crack tip is surrounded by a larger transformed zone associated with the larger applied stress, and the crack- growth rate is observed to decrease sharply. As the crack grows out of this 
large transformed zone to the smaller transformed zone associated with an applied stress of 8.5 MPa $\sqrt{m}$ the rate of cruck growth is observed to increase. A large zone associated with the application of a single overload of $12.3 \mathrm{MPa} \sqrt{m}$ caused a rapid decrease in the crack-growth rate followed by a gradual increase in growth rate as the crack tip moved out of the large zone. Changing $\mathrm{K}_{\min }$ without changing $\mathrm{K}_{\text {mer }}$ does not appear to change the crack-growth rate or the size of the transformed zone.

\section{SUMMARY}

The applicability of Raman spectroscopy to materials - and to the characterization questions raised by high-technology, hibh-temperature and energy-related materials - has been extended by recent advances in photon detectors. The utilization of two-dimensional array detectors for Raman spectroscopy has increased the sensitivity of the technique by virtually eliminating the detector dark count as a source of noise and has resulted in the development of a new method for obtaining multiplexed, spatially- resolved Raman spectra. By using the spatial resolution of the detector to achieve spatial resolution at the sample, spectra from many spatially resolved elements at the sample can be collected and analyzed simultaneousiy. This simultaneous multiplexing of both wavelength and position makes use of the inherent low dark count per pixel of the detecior and results in the rapid determination of a chemical composition profile. By translating the sample (in one direction) across the illuminating slit-like beam a map of the chemical composition of the sample surface can be determined. This technique for chemical mapping can be adopted to most forms of optical spectroscopy when an appropriate two-dimensional detector is used. This pafer has illustrated some of these capabilities by describing results from on-going work on carbon films and fibers and on phase- stabilized airconia ceramics.

\section{ACKNOWLEDGMENTS}

This work was supported by the Director, Office of Energy Research, U.S. Department of Energy, at Lawrence Berkeley Laboratory under contract DE-AC-03-76SF00098. The authore wish to thank the following people and institutions: Prof. IK.E. 
Spear of the Pennsylvania State University for supplying tĩe diamond thin film, Prof. Fitzer and Dr. Kunkele of the Institute fur Chemische Technik der Universitat Karlsruhe for supplying the carbon fibers, Dr. D. Marshall of Rockwell International for supplying the partially stabilized zirconia, and Komag Inc. and Lin-data Co for supplying the rigid carbon-covered disks. The studies of carbon overcoats on rigid disks were in collaboration with Prof. D. Bogy of the University of California, Berkeley and Dr. H.-c Tsai, Dr. M.K. Kundmann, Dr. M.R. Hilton, and Dr. S.T. Mayer. The studies of transformed zones in partially stabilized zirconia were in collaboration with Prof. R.O. Ritchie and Dr. R.H. Dausleardt of the Lawrence Berkeley Laboratory and the Univeristy of Califurnia, Berkeley. 


\section{REFERENCES}

1. A.G. Evans and A.H. Heuer, J. Am. Ceram. Soc. 63, 241 (1980).

2. L. Soto and F. Adar, Microbeam Analysis-1984, A.D. Romig, Jr. and J.I. Goldstein, Eds., San Francisco Press, 1984, p.121; P.V. Huong, P. Boutinaud, S. Kassoui, and A. Leycuras, Microbeam Analysis. 1984, D.E. Newbury, Ed., San Francisco Press, 1988, p.167 and others in that volume.

3. D.K. Veirs, V.K.F. Chia, and G.M. Rosenblatt, App. Opt. 26, 3530 (1987).

4. D.B. ChasE, J. Am. Chem. Soc. 108, 7485 (1986); C.G. Zimba, V. Hallmark, J.D. Swalen, and J.F. Rabolt, Appl. Spec. 41, 761 (1987).

5. K.E. Spear, J. Am. Ceram. Soc. 72, 171 (1989); R. Messier, A. Badzian, T. Badzian, K.E. Spear, P. Bachmann, and R. Roy, Thin Solid Films 153, 1 (1987).

6. H.-c. Tsai, D.B. Bogy, M.K. Kundmann, D.K. Veirs, M.R. Hilton, and S.t. Mayer, J. Vac. Sci. Technol. A 6, 2307 (1988).

7. F. Tuinstra and J. L. Koenig, J. Chem. Phys. 53, 1126 (1970).

8. C. Beny-Bassez and J.N. Rouzaud, Scan. Elec. Micros. 1985, 119 (1985).

9. C.A. Johnson, J.W. Patrick and K.M. Thomas, Fuel 65, 1284 (1986).

10 D. Beeman, J. Silverman, R. Lynds and M.R. Anderson, Phys. Rev. B 30, 870 (1984); M. DiDomenico, Jr., S.H. Wemple, S.P.S. Porto and R.P. Bauman, Phys. Rev. 174, 522 (1968).

11. A. Richter, H.-J. Scherbe, W. Pombe, D.-W. Brzezinka, and I. Muhling, J. Non-Crystal. Sol. 88, 131 (1986).

12. R.J. Day, I.M. Robinson, M. Zakikhani, and R.J. Young, Polymer 28, 1833 (1987).

13. R.H. Dauskardt, W. Yu and R.O. Ritchie, J. Am. Ceram. Soc. 70, C248 (1987)

14. R.H. Daushardt, D.K. Veirs, and R.O. Ritchie, J. Am. Ceram. Soc. in press. 15. D.R. Clarke and F. Adar, Advances in Materials Characterization, D.R. Rossington, R.A. Condrate, and R.L. Snyder, Eds., Plenum, p. 199, 1983.

16. R.H. Dauskardt, D.B. Marshall and R.O. Ritchie, Proceedings of the Materials Research Society International Meeting, 1988. 
Table I: Positions, linewidths, and intensity ratios of the graphite and disorder induced Raman bands of carbon thin films obtained from fitting the observed data to a theoretical model. The percentage of $\mathrm{sp}^{3}$ bonding is obtainied from the shift of the G-band.

\begin{tabular}{|c|c|c|c|c|c|c|c|}
\hline \multirow[b]{2}{*}{ Sample } & \multirow[b]{2}{*}{$\begin{array}{c}\text { Anneal } \mathrm{T} \\
{ }^{\circ} \mathrm{C}\end{array}$} & \multicolumn{2}{|c|}{ G-BAND } & \multicolumn{2}{|c|}{ D-BAND } & \multirow[b]{2}{*}{$I_{d} / I_{g}$} & \multirow[b]{2}{*}{$\% \mathrm{sp}^{3}$} \\
\hline & & $\begin{array}{c}\text { Position } \\
\mathrm{cm}^{-1}\end{array}$ & $\begin{array}{c}\text { Linewidth } \\
\mathrm{cm}^{-1}\end{array}$ & $\begin{array}{c}\text { Position } \\
\mathrm{cm}^{-1}\end{array}$ & $\begin{array}{c}\text { Linewidth } \\
\mathrm{cm}^{-1}\end{array}$ & & \\
\hline K1 & - & 1562 & 176 & 1393 & 347 & 1.47 & $\mathbf{5}$ \\
\hline K5 & 350 & 1571 & 160 & 1391 & 349 & 1.72 & 2 \\
\hline $\mathrm{K} 2$ & 450 & 1575 & 140 & 1386 & 335 & 2.16 & 0 \\
\hline L3 & - & 1575 & 159 & 1372 & 300 & 1.54 & 0 \\
\hline
\end{tabular}


Table II: Intensity ratios, domain sizes, line positions and linewidths of Raman peaks from carbon fibers annealed at $2400^{\circ} \mathrm{C}, 2600^{\circ} \mathrm{C}$, and $2800^{\circ} \mathrm{C}$.

\begin{tabular}{|c|c|c|c|c|c|c|}
\hline & \multirow[t]{3}{*}{$I_{d} / I_{g}$} & \multirow[t]{2}{*}{$\mathrm{L}_{a}$} & \multicolumn{2}{|c|}{ G-BAND } & \multicolumn{2}{|c|}{ D-BAND } \\
\hline & & & Position & Linewidth & Position & Linewidth \\
\hline & & $\mathrm{nm}$ & $\mathrm{cm}^{-1}$ & $\mathrm{~cm}^{-1}$ & $\mathrm{~cm}^{-1}$ & $\mathrm{~cm}^{-1}$ \\
\hline CF2400 & 0.63 & 6.7 & 1565.6 & 38.6 & 1348.8 & 38.2 \\
\hline CE2600 & 0.48 & 8.3 & 1572.8 & 32.0 & 1353.1 & 29.0 \\
\hline CF2800 & 0.28 & 12.5 & 1570.3 & 29.4 & 1354.3 & 31.6 \\
\hline
\end{tabular}




\section{FIGURE CAPTIONS}

FIGURE 1: A single sharp Raman peak at $1332 \mathrm{~cm}^{-1}$ is a distinguishing feature of diamond thin films. The weak, broad peaks extending from $1100-1600 \mathrm{~cm}^{-1}$ are due to amorphous carbon which has a much larger Raman cross-section.

FIGURE 2: Raman spectra of the carbon overcoats on magnetic media disks from two different manufacturers. Samples K1, K5, and K2 were sectioned from the same disk and annealed at $30^{\circ} \mathrm{C}, 350^{\circ} \mathrm{C}$, and $450^{\circ} \mathrm{C}$ respectively. The data and fit, comprised of two peaks and a background (not shown), are shown for the four samples.

FIGURE 3: The effect of annealing on single carbon fibers is seen in the Raman spectra. At higher annealing temperatures the G-line at $1575 \mathrm{~cm}^{-1}$ grows at the expense of the D-line at $1350 \mathrm{~cm}^{-1}$ and the linewidths decrease. This is an indication of graphite microcrystallite growth from 7 to $13 \mathrm{~nm}$.

FIGURE 4: The tetragonal and monoclinic phases of zirconia have Raman peaks at different frequencies which can be used to determine the relative amounts of each phase. In this work the $181,192 \mathrm{~cm}^{-1}$ pair is used to measure the monoclinic phase and the $264 \mathrm{~cm}^{-1}$ peak is used to measure the amount of tetragonal phase.

FIGURE 5: The crack growth rate and applied stress (schematic) in toughened PSZ are shown as a function of crack extension for a fatigue-crack grown under varying amplitude cyclic loads.

FIGURE 6: A map of the monoclinic phase fraction of the zirconia specimen whose applied stress and crack growth rate are shown in Fig. 5 indicates that the stress history of the material is revealed in the extent and degree of transformation of the transformed zone. Large stresses induce a larger transformed zone around the crack tip that remains after the crack tip moves forward. 


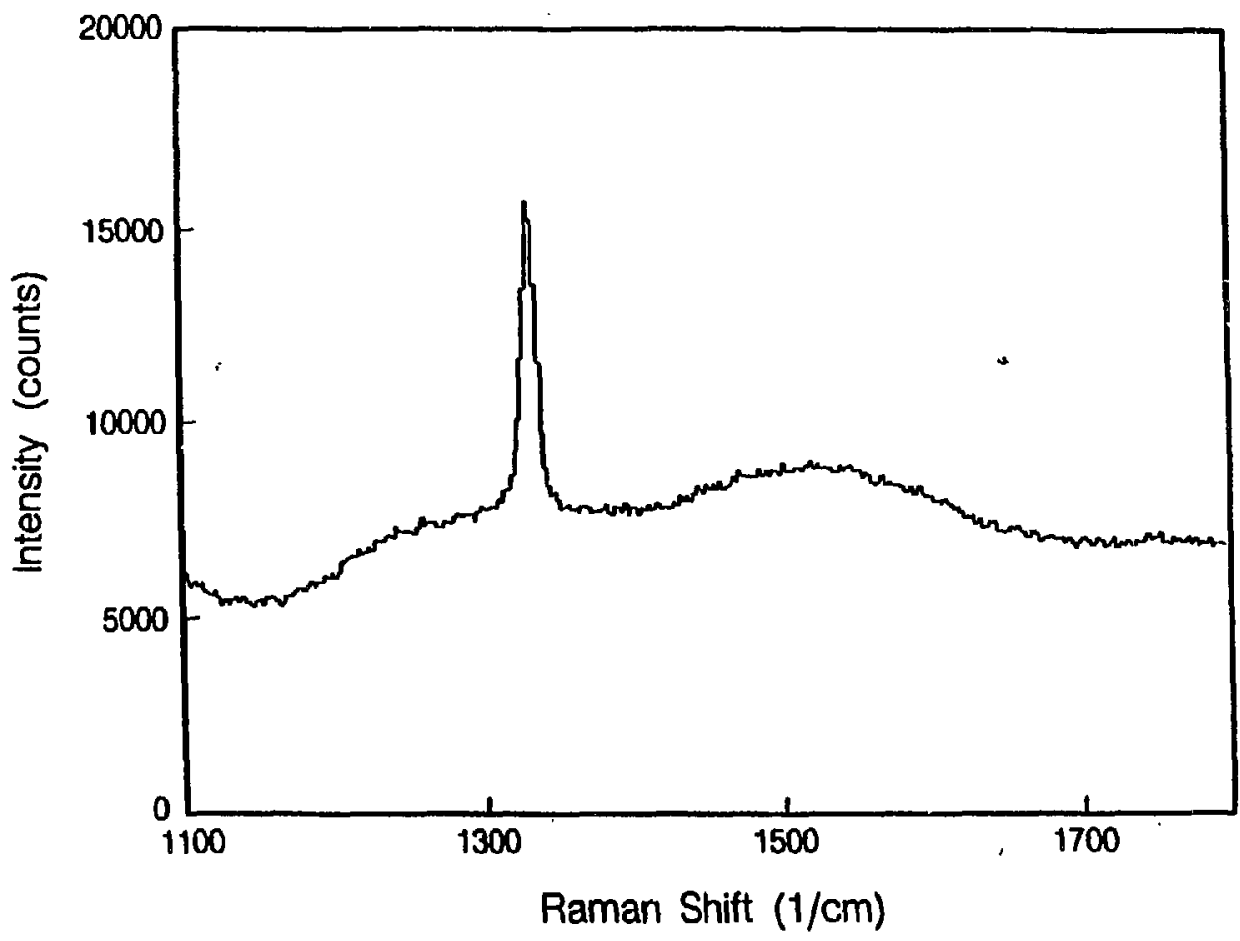

XBL 887.8923

Fig. 1 


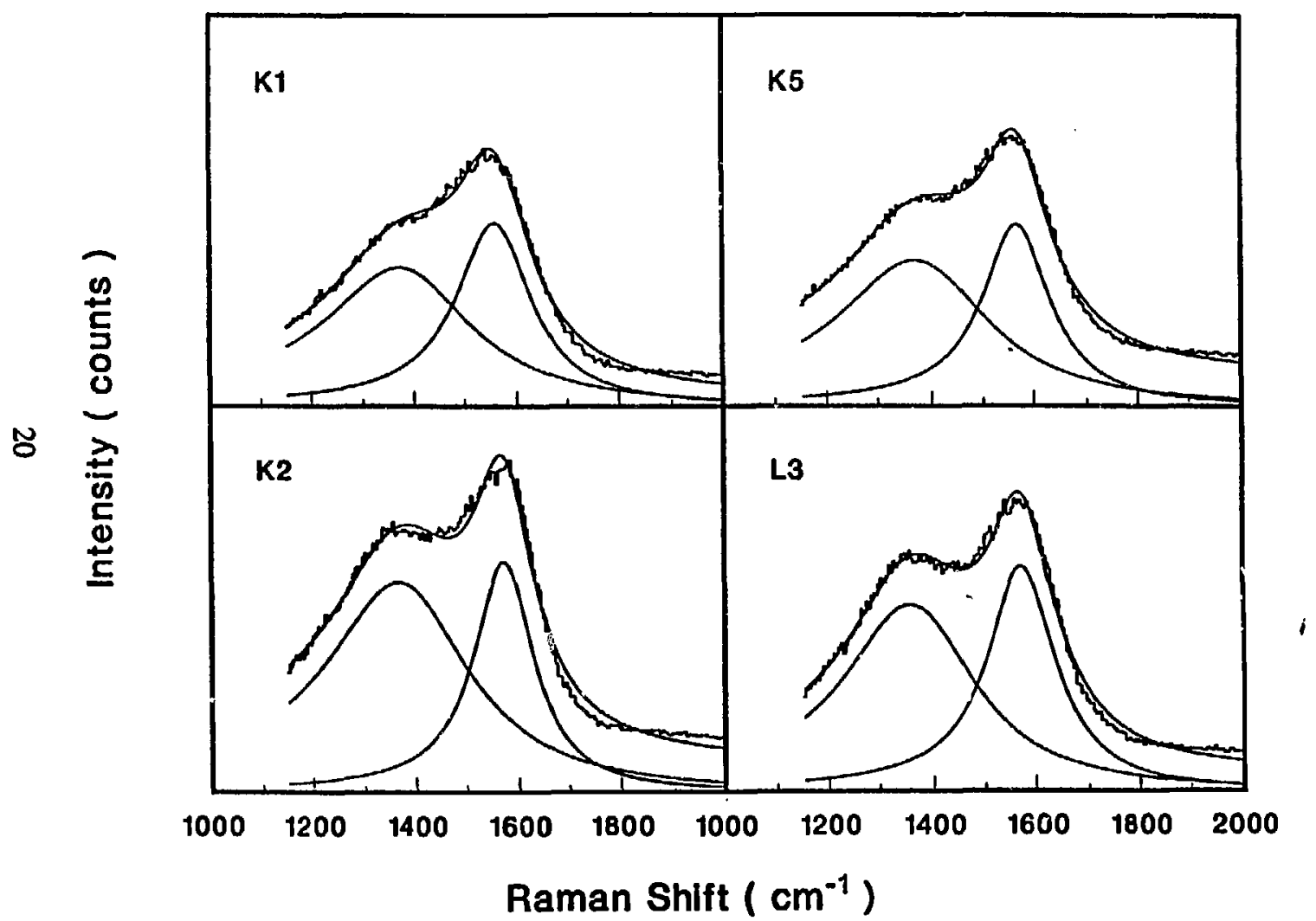

Fig. 2 


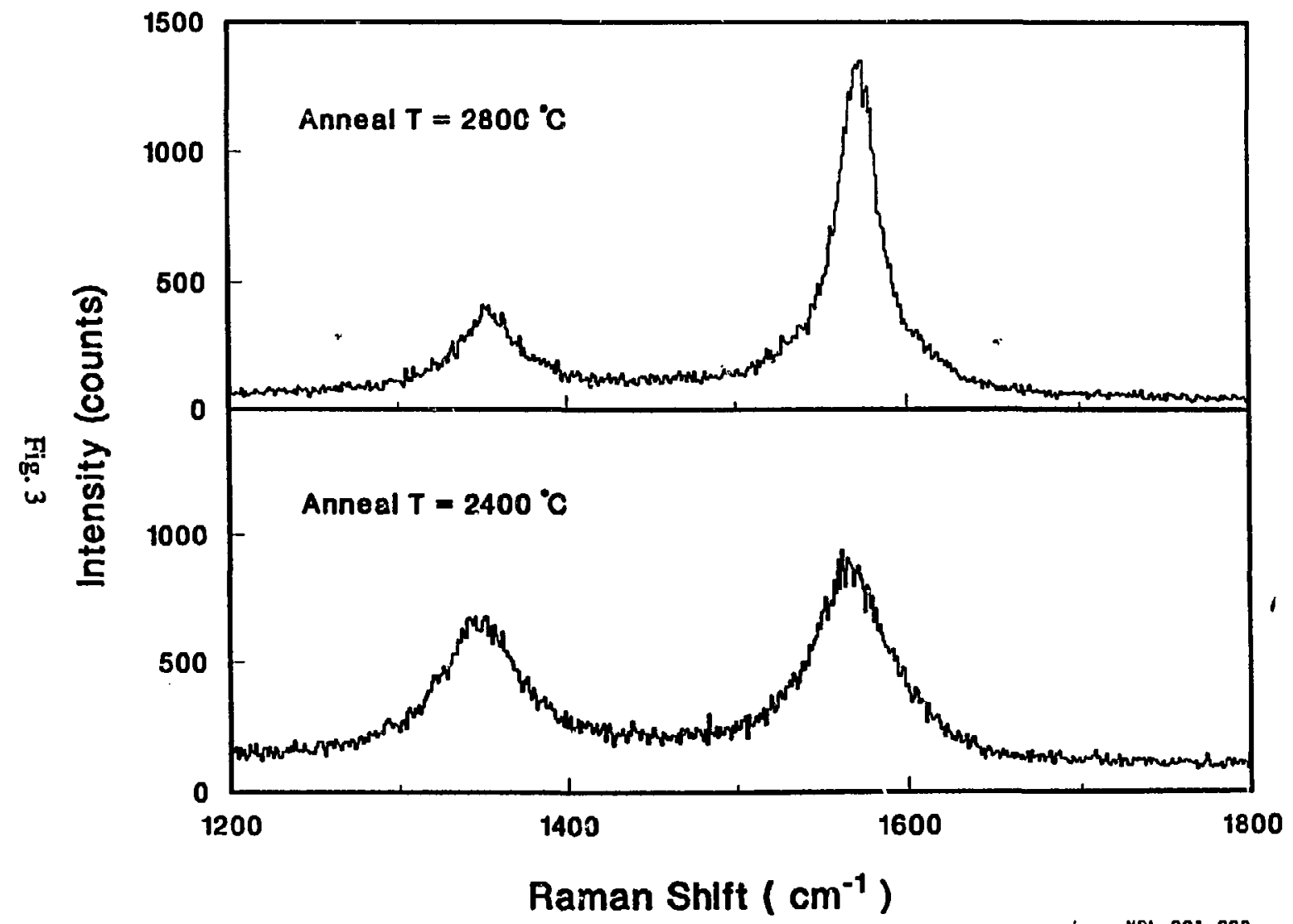

XBL 891-223 


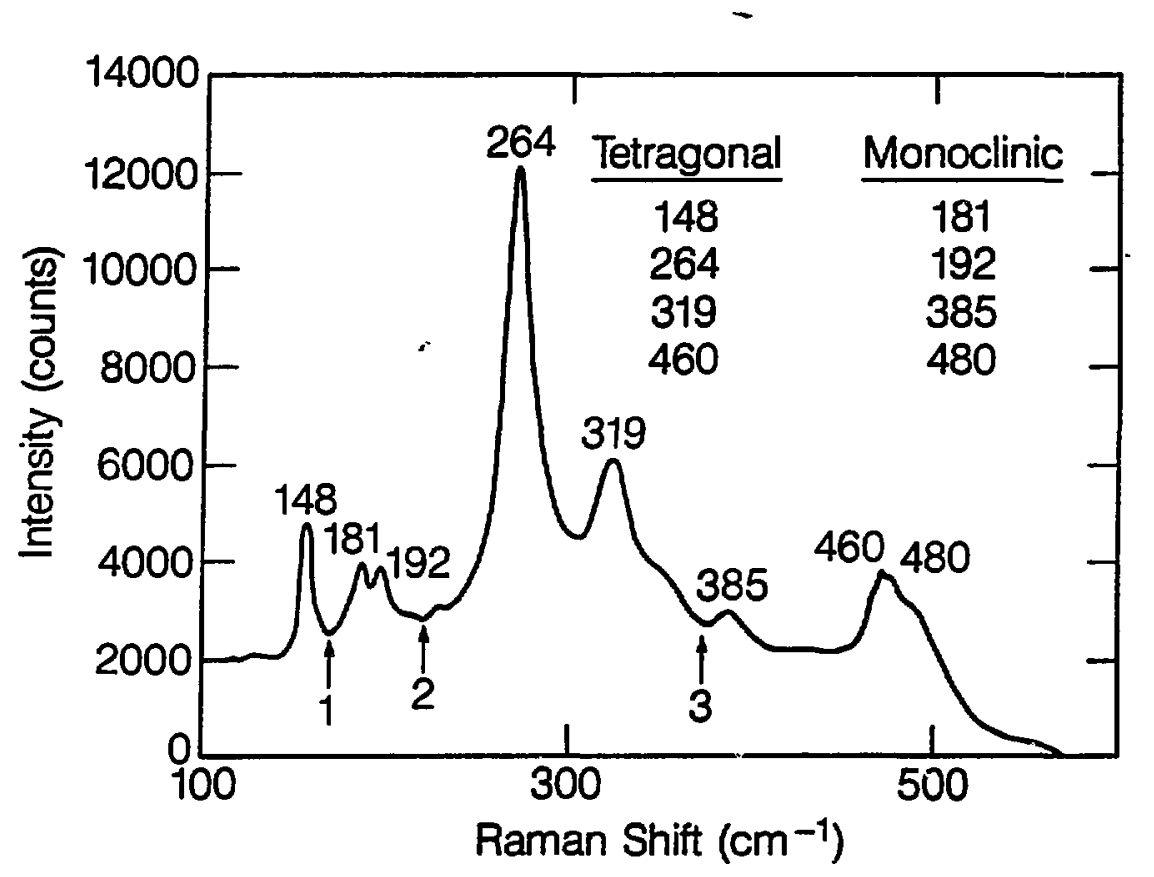

XBL $883-6010$

Fig. 4 


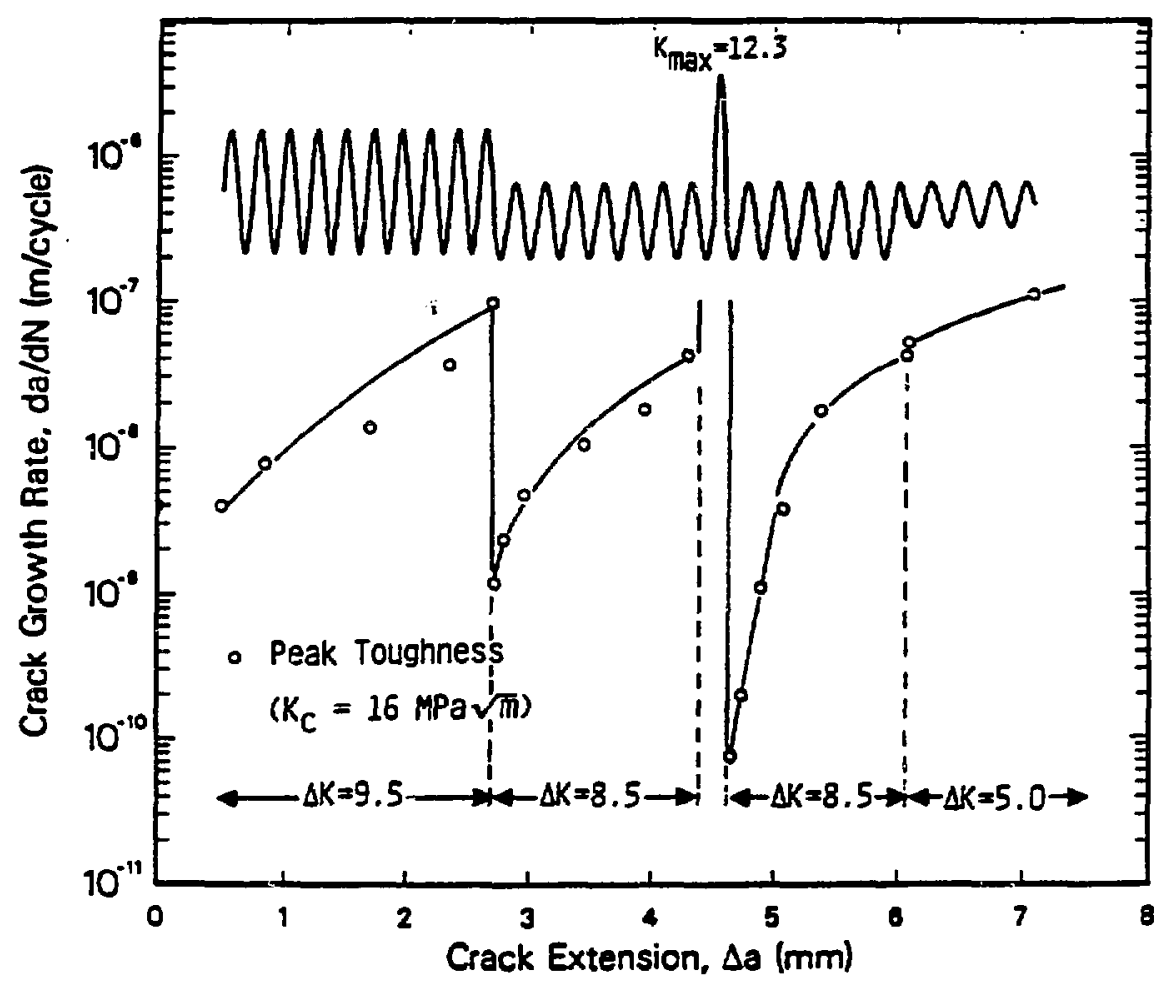

XBL 885-1877A

Fig. 5 


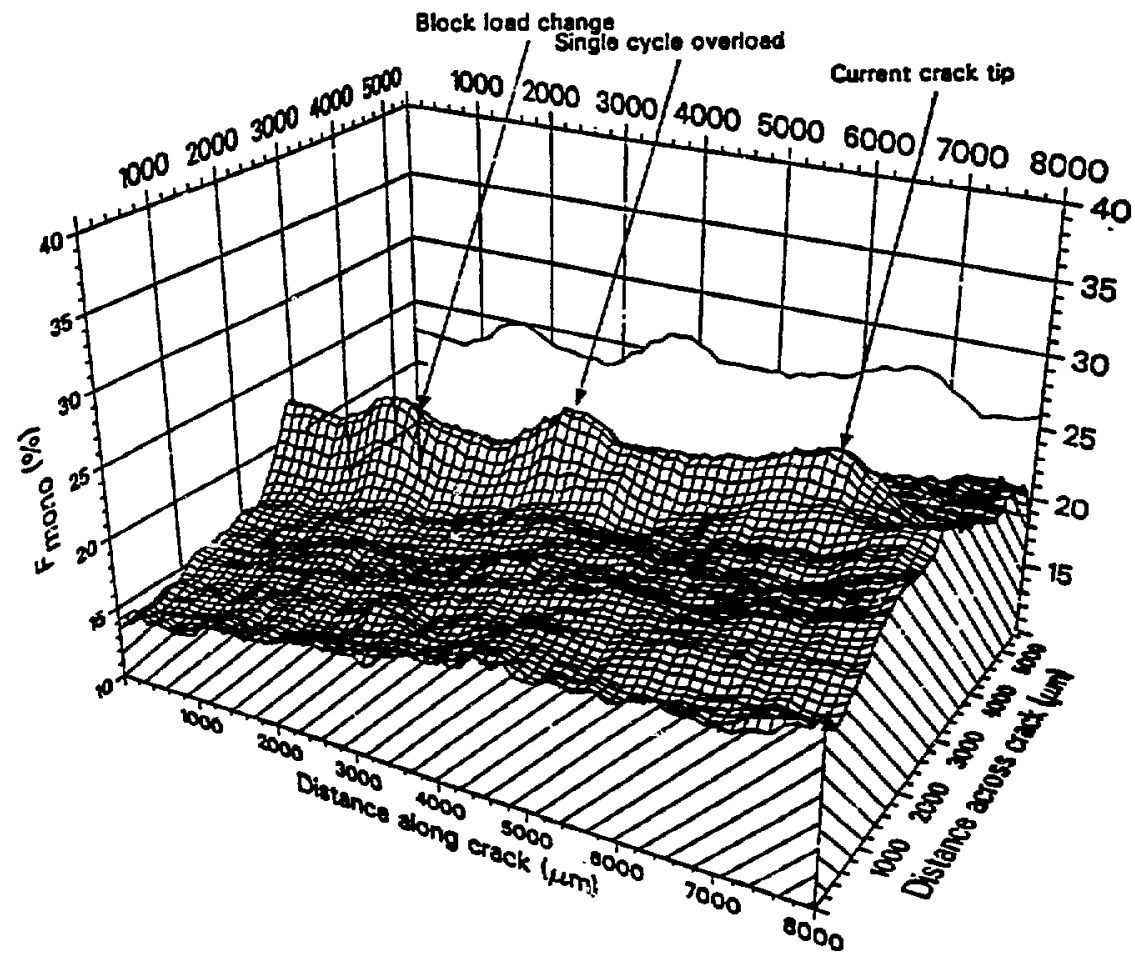

xาc $591-220$

Fig. 6 